\title{
Surfaces with Polar Structure
}

\author{
K. Karčiauskas and J. Peters
}

April 28, 2006

\begin{abstract}
We describe the structure and general properties of surfaces with polar layout. Polar layout is particularly suitable for high valences and is, for example, generated by a new class of subdivision schemes. This note gives an high level view of surfaces with polar structure and does not analyze particular schemes.
\end{abstract}

\section{Introduction}

One way to introduce polar surface layout is by comparison with subdivision schemes such as Catmull-Clark. Subdivision surfaces can be characterized as a
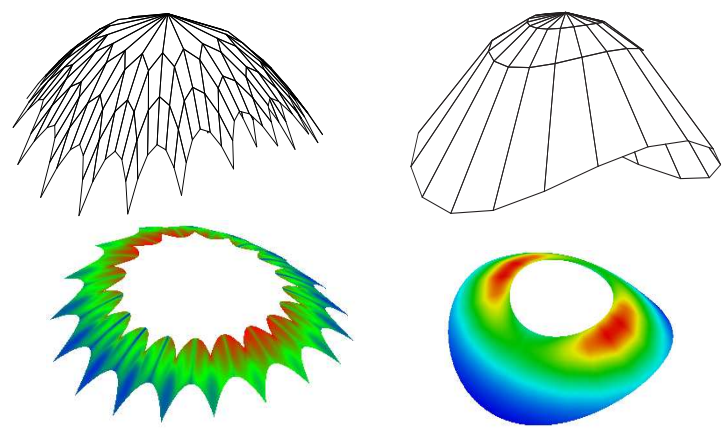

Figure 1: (top) Control net with central node of valence 18 positioned on the paraboloid $z=-x^{2}-0.6 y^{2}$. (left) A ring with sprocket structure. (right) A ring with polar structure. (bottom) Surface rings shaded by Gauss curvature generated by (left) bicubic surface ring and (right) polar surface ring [4].

sequence of nested spline rings converging to an extraordinary point (see e.g. [7]; 

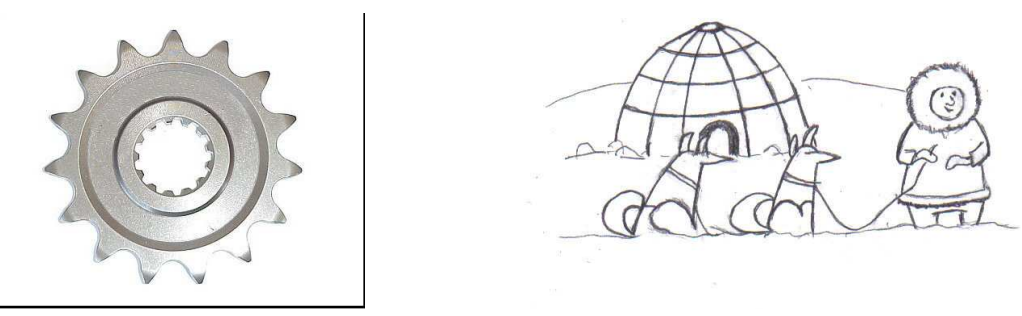

Figure 2: (left) A sprocket. (right) A polar structure seems natural in some settings.

the alternative view, the refinement of a control structure, is discussed in Section 3). The natural layout of such a spline ring near an extraordinary vertex of high valence is shown in Figure 1, right, and is quite unlike the standard subdivision ring shown in Figure 1, left: in standard subdivision, the spline rings are bounded by $n$ spline curve pieces joining with sharp angles (e.g. Catmull-Clark subdivision [1], Doo and Sabin's subdivision [2] or Loop's subdivision [5]). The $n$ corners of the rings then induce a shape similar to a sprocket (see Figure 2, left). We note that, as the valence $n$ increases, the length of the inner and hence outer boundary of a sprocket ring increase fast, due to the corners. - By contrast, a spline ring with polar structure is bounded by a single spline curve consisting of $n$ smoothly connected pieces. Polar surface rings therefore mimic the layout of architectural domes (Figure 2, right) and spider webs.

A second way to motivate polar surface structure is to observe that sprocket subdivision algorithms typically yield poor shape (see e.g. Figure 3) when the
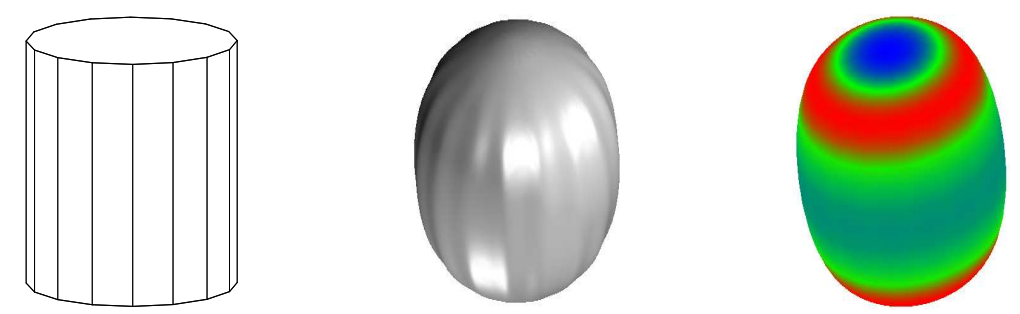

Figure 3: (left) A 16-sided cylinder, (middle) Catmull-Clark interpretation of the 16-sided cylinder, (right) Gauss-curvature shaded interpretation of the cylinder according to polar guided subdivision [4]. 

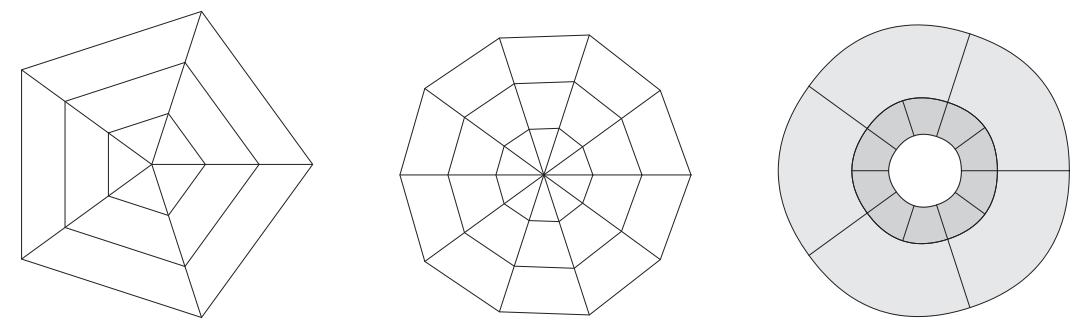

Figure 4: (left) Input polar design net. (middle) Doubling of valence by uniform cubic knot insertion applied to each layer separately. (right) Transition with virtual T-corners.

valence is high. By contrast, in the polar layout, increasing the valence is as easy as knot insertion in the circular layers and therefore allows for little or no change in the shape of the ring. In fact, the valence can be varied from step to step by reinterpreting a ring as consisting of more pieces as in Figure 4, right. For polar subdivision schemes, increasing the valence is often desirable, because (i) if there is an underlying guide surface as in [4], increased valence can improve approximation order and capture shape better, and (ii) increasing valence avoids special treatment of low valences as is often needed to improve subdivision with standard layout.

While the two introductory characterizations focused on surface properties, these properties are the result of surface partition inherited from a partition of its domain. We discuss this formal difference between standard sprocket and patch layout next.

\section{Domain Layout}

Subdivision surfaces can be characterized as consisting of sequences of nested spline rings converging to extraordinary points. For an extraordinary point of valence $n$, each spline ring is defined on a domain consisting of $n$ segments. Each segment is a copy of a standard compact domain and the edges are identified to give the topological structure of an annulus.

Formally, near an extraordinary or central point $\mathrm{x}^{\infty}$, a subdivision surface 

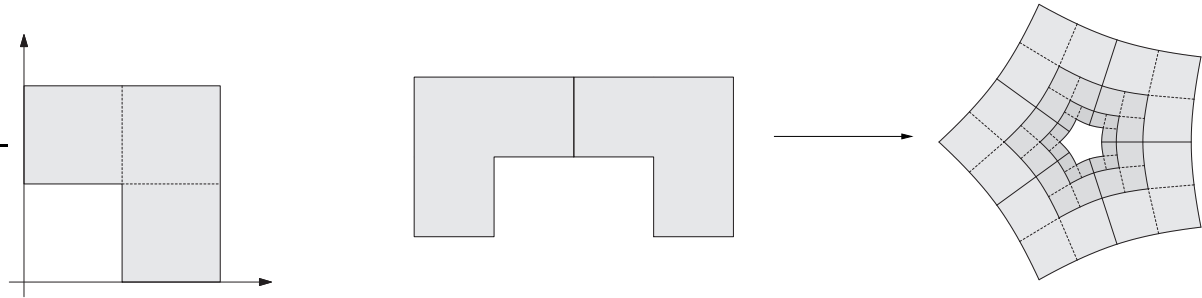

Figure 5: Sprocket layout. (a) Basic domain $\Sigma$, (b) joining copies of $\Sigma$ to form $\mathbf{S}$ and (c) polar layout.

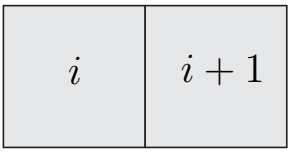

(b)

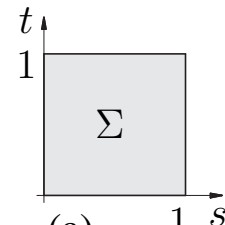

(a)

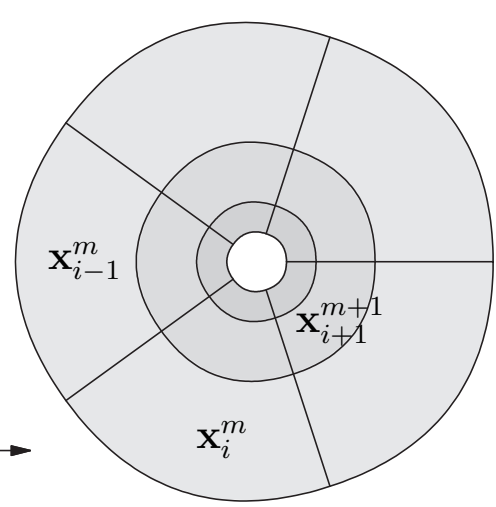

(c)

Figure 6: Polar layout. (a) Basic domain $\Sigma$, (b) joining copies of $\Sigma$ to form $\mathbf{S}$ and (c) polar layout.

$\mathbf{x} \in \mathbb{R}^{d}$ is the union of a sequence of nested surface rings $\mathbf{x}^{m}$ contracting to $\mathrm{x}^{\infty}$ :

$$
\mathbf{x}=\mathbf{x}^{\infty} \cup \bigcup_{m \in \mathbb{N}} \mathbf{x}^{m} .
$$

Each surface ring $\mathbf{x}^{m}$ is in turn a union of $n$ segments $\mathbf{x}_{i}^{m}, i=0, \ldots, n-1$,

$$
\mathbf{x}^{m}=\bigcup_{i=0}^{n-1} \mathbf{x}_{i}^{m}
$$

where a segment $\mathbf{x}_{i}^{m}$ can, for example, consist of several smoothly connected Bézier patches. The ring-like structure of $\mathbf{x}^{m}$ is the result of the periodicity of the domain $\mathrm{S}$, composed of $n$ copies of a basic domain $\Sigma \subset \mathbb{R}^{2}$ :

$$
\mathbf{S}:=\Sigma \times \mathbb{Z}_{n}, \quad \mathbb{Z}_{n}:=\mathbb{Z} \bmod n .
$$


Standard-sprocket and polar subdivision differ in the basic domain $\Sigma$ and in the way the edges of consecutive segments $(\Sigma, i)$ and $(\Sigma, i+1)$ are set equal to form a joint domain. Figure 2 shows the standard, say Catmull-Clark, setting. The basic domain is $\Sigma:=[0,2]^{2} \backslash[0,1)^{2}$. To topologically join the basic domains, always an edge on the $s$-axis is set equal to an edge on the $t$-axis of the neighbor's basic domain. Consecutive rings then have the layout familiar from the characteristic map of Catmull-Clark subdivision and shown in Figure 2 (c).

In the polar layout, the basic domain $\Sigma$ is simply the unit square. Opposing, rather than adjacent, edges are set equal for neighboring pieces to form the domain. The polar surface is therefore a cyclic strip of spline patches as shown in Figure 2 (c). Such a spline strip affords a change of valence by knot insertion or knot removal - as is not possible in the sprocket setting. For example, by uniform knot insertion in the 'circular' direction, one can reinterpret a spline ring as corresponding to valence $2 n$ if the original valence was $n$.

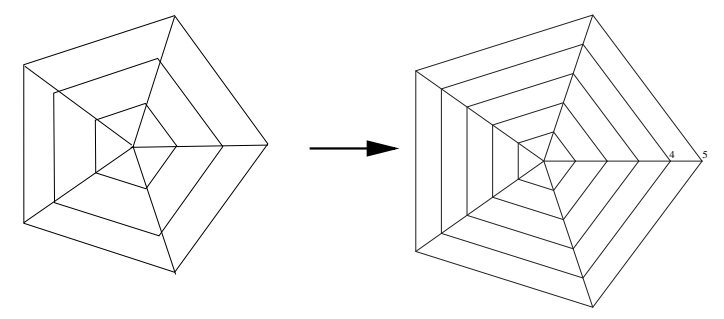

Figure 7: Refinement of a polar net near an extraordinary node.

\section{Design control net and recursive display}

For standard subdivision schemes a network of line segments connecting control points is an important feature and is often taken as a proxy for the subdivision limit surface. In fact, this net plays two roles. First, it serves as a design net to adjust the shape of the surface and secondly, the recursively refined nets serve for display. Polar design nets are well-defined and consist of extraordinary nodes, i.e. nodes surrounded by triangles, and of quadrilaterals with nodes of valence four away from the global boundary. With extraordinary nodes so defined, there is no 'regular' valence. There is also no obvious refined control net suitable for display. Each triangle is subdivided into one quadrilateral and a smaller triangle (see Figure 2). The control net near the extraordinary node therefore increases density 
only in the radial direction leading away from the extraordinary node. This nonrefinement in the circular direction distinguishes polar subdivision from singular tensor-product schemes such as [6]. The valence at the centralpoint for tensorproduct surfaces with pinch points truly increases to infinity. So, if one retains standard tensor-product spline refinement in both parameters for the quadrilateral facets and uni-directional refinement near the extraordinary point, T-corners appear. These T-corners are virtual in that they do not correspond to a join of three patches but to the transition between two patches where one is represented in subdivided form. Virtual T-corners also appear when the valence is doubled (see Figure 4). Instead of the refined mesh, for polar subdivision it is natural to just show the sequence of surface rings.

\section{Switching from standard to polar layout}

There is no theoretical difficulty in locally converting standard meshes to polar meshes. Five-sided regions or, equivalently, new extraordinary points of valence five allow transition from standard subdivision to polar subdivision (Figure 8, left) as follows. If one places a circle inside an $n$-gon and connects the midpoints of the $n$-gon edges to the circle (cf. Figure 8, middle), all newly created extraordinary points or auxiliary regions are of valence five with combinatorial axial symmetry, as shown in Figure 8, right. Nevertheless, polar subdivision should not be forced on all of a mesh with a standard structure but should be applied where it naturally occurs, i.e. in many-sided blends and to cap off cylindrical shapes.

Typically, standard layout makes fair design in high-valence standard configurations difficult (see Figure 1). Auxiliary five-sided regions allow combining, say
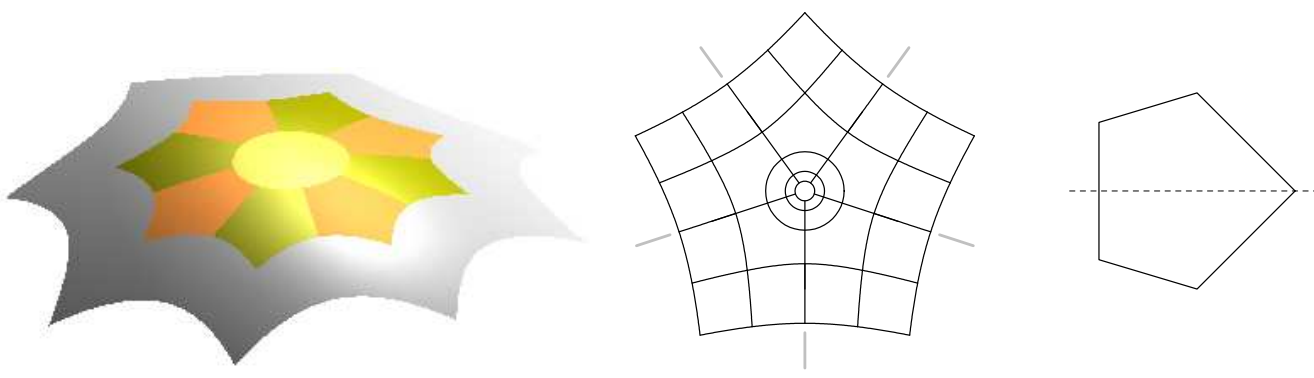

Figure 8: Switching from standard to polar layout via pentagonal patches. 
Catmull-Clark meshes with a polar generalization of uniform bicubic splines [3]. From this point of view, polar subdivision is an addition to, not a replacement for, the standard subdivision. Of course, there are also meshes that have entirely polar structure; see Figure 10. Such meshes have the combinatorial structure of objects of revolution with one or two poles.

\section{Conclusion}

Intentionally, to keep the focus, this note gives only an overview, and does not describe specific schemes. Their definition and analysis $[4,3]$ will round out the picture as their publication status advances.

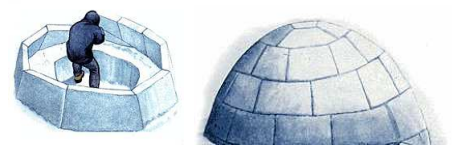

Acknowledgement: This work was supported by NSF Grants DMI-0400214 and CCF-0430891. Figure 9: A specific scheme We thank U. Reif and C. de Boor for feedback on to build a polar structure. an earlier draft.

\section{References}

[1] E. Catmull and J. Clark. Recursively generated B-spline surfaces on arbitrary topological meshes. Computer Aided Design, 10:350-355, 1978.

[2] D. Doo and M. Sabin. Behaviour of recursive division surfaces near extraordinary points. Computer Aided Design, 10:356-360, September 1978.

[3] K. Karčiauskas and J. Peters. A bicubic polar subdivision scheme. submitted.

[4] K. Karčiauskas and J. Peters. Concentric tesselation maps and guided surface rings. Computer Aided Geometric Design, x(x):xx-xx, 200x. under review, original title: Guided subdivision.

[5] Charles T. Loop. Smooth subdivision surfaces based on triangles, 1987. Master's Thesis, Department of Mathematics, University of Utah.

[6] Géraldine Morin, Joe D. Warren, and Henrik Weimer. A subdivision scheme for surfaces of revolution. Computer Aided Geometric Design, 18(5):483$502,2001$. 

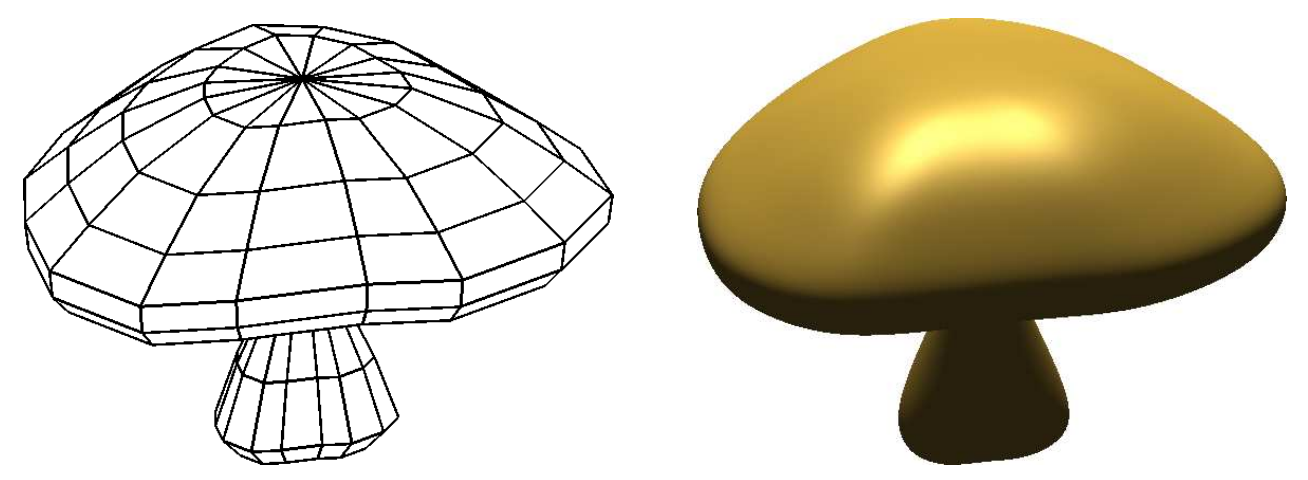

Figure 10: Mesh with polar structure and corresponding surface.

[7] U. Reif and J. Peters. Topics in multivariate approximation and interpolation. In K. Jetter et al., editor, Structural Analysis of Subdivision Surfaces - A Summary, pages 149-190. Elsevier Science Ltd, 2005. 